\title{
Optical coherence tomography findings in compressive optic neuropathy and pre-existing glaucoma
}

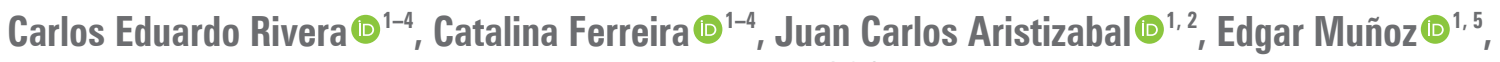 \\ Ankur Seth $\mathbb{D}^{1,3,6}$ \\ ${ }^{1}$ Collective Innovations Colombia, Cali, Colombia \\ ${ }^{2}$ Pontificia Universidad Javeriana, Cali, Colombia \\ ${ }^{3}$ GSR Medical Center, Cali, Colombia \\ ${ }^{4}$ GSR International, United States \\ ${ }^{5}$ UT Health San Antonio, San Antonio, United States \\ ${ }^{6}$ University of Tennessee Health Science Center, United States
}

\begin{abstract}
BACKGROUND: We present the optical coherence tomography (OCT) findings in macular ganglion cell complex (GCC) and retinal nerve fiber layer (RNFL) in a case of a female patient with craniopharyngioma and preexisting glaucoma.

CASE PRESENTATION: 80-year-old female patient with a history of successfully suprasellar resection of craniopharyngioma performed eight years earlier and preexisting primary open-angle glaucoma treated with latanoprost indicated a one-month history of decreased vision in the left eye. The visual field showed a vertical hemifield defect in the right eye and an inferior arcuate defect in the left eye. A cerebral magnetic resonance image confirmed a new suprasellar tumor. The patient was successfully operated on one week after diagnosis. Visual acuity in her left eye improved substantially after surgery.

RESULTS: Optical coherence tomography of macular and RNFL showed thinning in the patient's right eye that corresponded with the vertical visual field defect. A "C" pattern that compromised the horizontal meridian differentiated from glaucoma that respects the horizontal meridian. The visual field showed a vertical hemifield defect in the right eye and an inferior arcuate defect in the left eye.

CONCLUSIONS: Optical coherence tomography is a non-invasive imaging procedure. It helps identify compression of the anterior visual pathways, resulting in progressive thinning of RNFL and macular ganglion cell complex (GCC). It has a good correlation with visual fields.
\end{abstract}

KEY WORDS: optical coherence tomography; glaucoma; craniopharyngioma; visual field; visual acuity

Ophthalmol J 2021; Vol. 6, 249-254

CORRESPONDING AUTHOR:

Carlos Rivera, Holguines Trade Center, Cra 10 11-60, office 236, tel: (2) 3165225961, (572) 5546567; e-mail: carlosriverahoyos@gmail.com, crivera@grupogsr.co 


\section{INTRODUCTION}

Chiasmal compression predominantly affects crossed nerve fibers associated with the nasal hemiretina, leaving uncrossed nerve fibers that originate in the temporal hemiretina relatively preserved [1]. Chiasmal compression is traditionally diagnosed by a characteristic temporal visual field (VF) defect along the vertical meridian.

Optical coherence tomography (OCT) is a non-invasive optical technique that allows in vivo cross-sectional imaging of the optic nerve head and the retina [2]. The thickness of the retinal macular layers is analyzed through cross-sectional images (B-scans) [3].

The inner retinal layers are the retinal nerve fiber layer (RNFL), ganglion cell layer (GCL), and inner plexiform layer (IPL). The GCL encompasses the cell bodies of the ganglion cells of the retina. The axons of the retinal ganglion cells form the RNFL that travel out of the eye in the optic nerve, chiasm, and tract and finally synapse in the lateral geniculate body before conveying their information to the visual cortex. Compressive lesions in the optic nerve, chiasm, or tract will manifest as thinning of the RNFL and GCL [4].

Optical coherence tomography evaluations of the RNFL and GCL can be performed through peripapillary RNFL thickness and macular cube
OCT images. They can help differentiate compressive optic neuropathies from glaucoma and other optic nerve neuropathies [5].

We present the OCT macular and RNFL findings in a case of a female patient with craniopharyngioma and preexisting glaucoma.

\section{CASE REPORT}

We present a case of an 80-year-old female patient with a one-month history of decreased vision in her left eye. The patient had a history of successfully suprasellar resection of craniopharyngioma eight years earlier, primary open-angle glaucoma treated with latanoprost. Visual field defect showed a vertical hemifield defect in her right eye and an inferior arcuate defect in her left eye. Cerebral magnetic resonance imaging confirmed the new appearance of the suprasellar tumor.

We diagnosed:

- in the right eye: best-corrected visual acuity (BCVA) - 20/30, mild cataract, open angles, intraocular pressure (IOP) $-14 \mathrm{~mm} \mathrm{Hg}$, cup/disc $(\mathrm{C} / \mathrm{D})$ ratio -0.3 ;

- in the left eye: BCVA - 20/70, mild cataract, open angles, IOP $-14 \mathrm{~mm} \mathrm{Hg}, \mathrm{C} / \mathrm{D}$ ratio -0.8 , superior and inferior rim thinning.

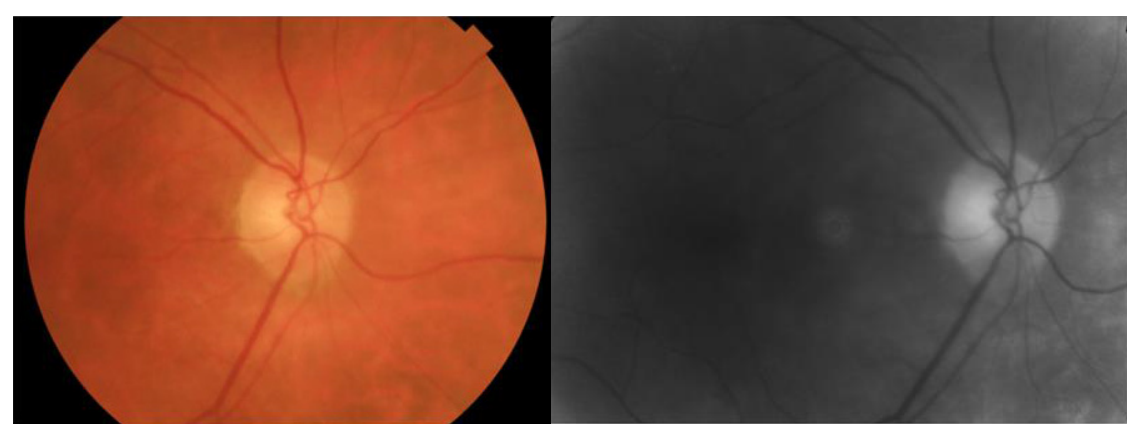

FIGURE 1. Right eye; A. Color optic nerve image; B. Red free image

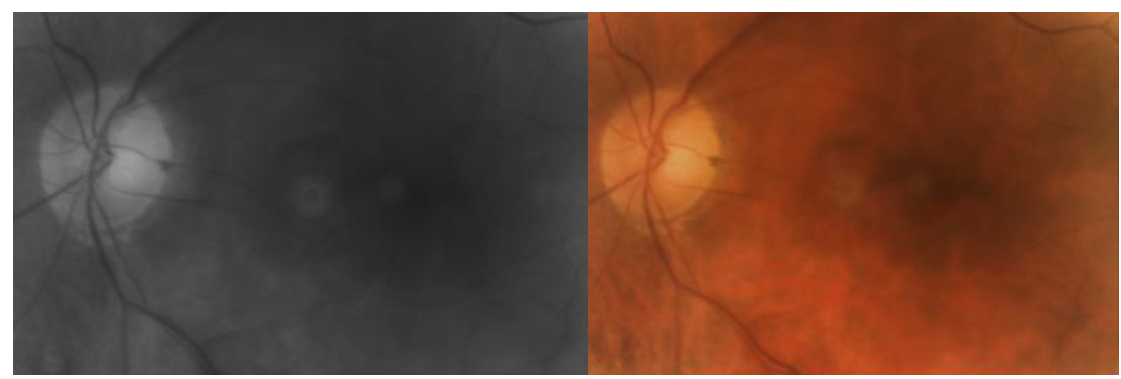

FIGURE 2. Left eye; A. Color optic nerve image; B. Red free image 
A

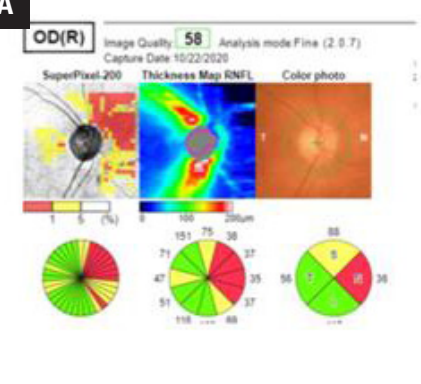

D
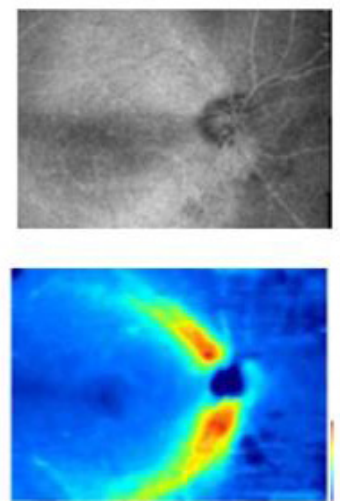

B

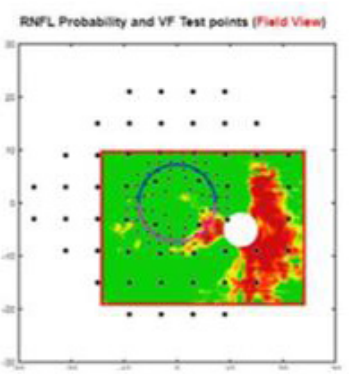

$\mathbf{E}$

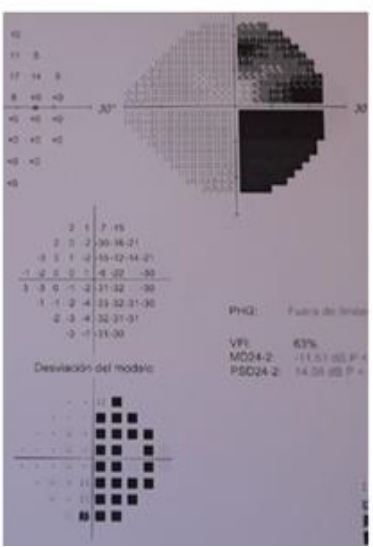

\section{c}
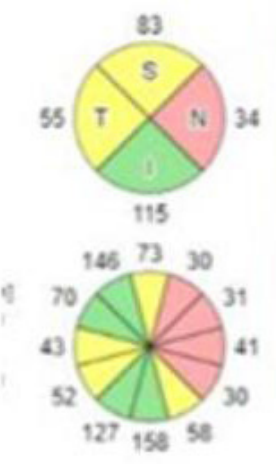

F

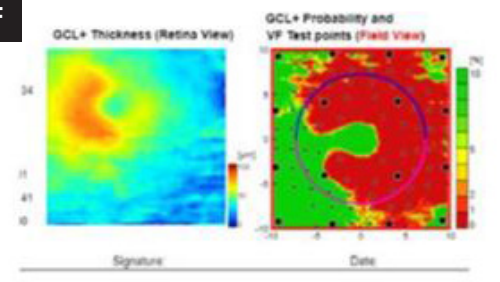

FIGURE 3. Right eye; A. RNFL deviation and clock hour meridian showing nasal thinning; B. RNFL probability and VF test points showing vertical temporal damage; C. RNFL clock hour meridian showing nasal thinning; D. Enface and thickness map showing nasal damage; E. Visual field showing temporal nasal defect; F. GCL thickness map showing "C" pattern compromising horizontal raphe. $\mathrm{GCL}$ — probability VF test points showing temporal damage

Optical coherence tomography findings in the right eye showed a nasal thinning in thickness, meridian, deviation, and enface map. RNFL and GCL probability maps showed a temporal defect that resembled a temporal visual field defect. All these findings were in the presence of a physiologic $\mathrm{C} / \mathrm{D}$ ratio.

These findings were typical of a compressive optic neuropathy.

Optical coherence tomography findings in the left eye showed superotemporal thinning in thickness, meridian, deviation, and enface map.

RNFL and GCL probability maps showed an inferior arcuate defect resembling the inferior arcuate defect present in the visual field. All these findings were correlated with a $\mathrm{C} / \mathrm{D}$ ratio of 0.8 with superior and inferior thinning. These findings were more compatible with glaucomatous optic neuropathy than compressive optic neuropathy.

The patient was successfully operated on one week after diagnosis. Visual acuity in her left eye improved substantially.

This case has a unique presentation where the right eye shows a physiological $\mathrm{C} / \mathrm{D}$ ratio in the presence of a vertical hemifield defect with a per- fect OCT correlation, typical of compressive optic neuropathy. On the other hand, the left eye showed a typical superior thinning with a corresponding inferior arcuate visual field defect and a very good OCT correlation, typical of glaucomatous neuropathy.

Visual acuity of the patient's left eye recovered to 20/30, the same level before surgery.

\section{DISCUSSION}

Compressive optic neuropathies typically present themselves

with slow, progressive, painless vision loss. Clinically it can help to differentiate them from other optic neuropathies [4]. The lesions most seen are meningiomas, craniopharyngioma, internal carotid or ophthalmic artery aneurysms and pituitary adenomas [6]. Reduced visual acuity, altered color vision, abnormal visual fields, pallor of the optic nerve are the clinical features of compressive optic neuropathies [7].

OCT findings include a "band atrophy" due to a more significant thinning of the horizontal sectors 
A

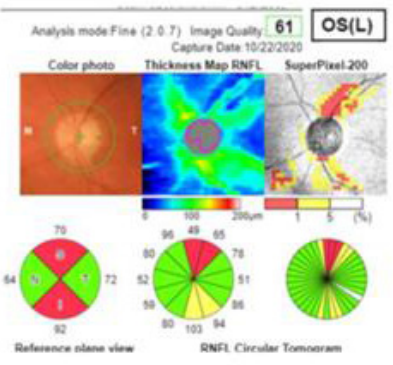

D
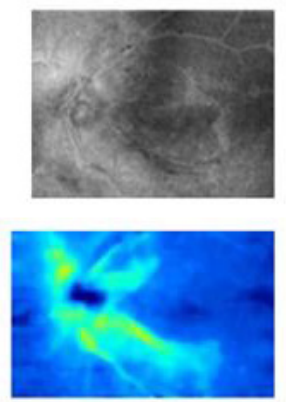

B

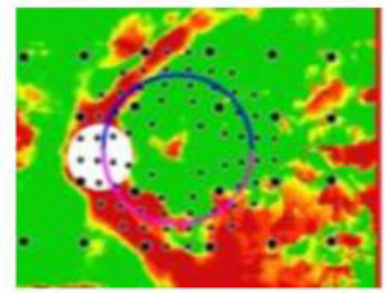

$\mathbf{E}$

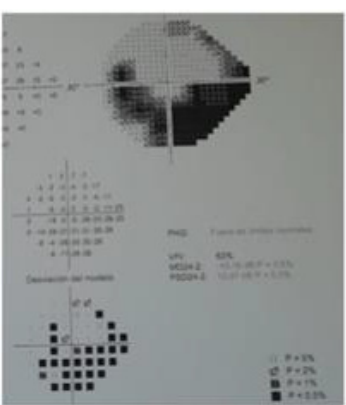

C
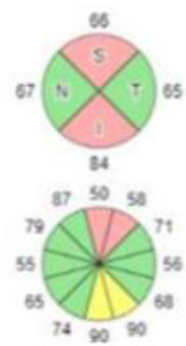

$\mathbf{F}$

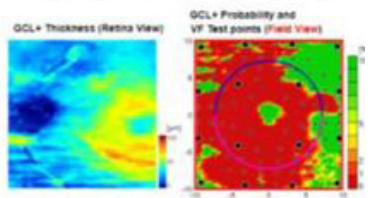

FIGURE 4. Left eye. A. RNFL deviation and clock hour meridian showing superotemporal thinning; B. RNFL probability and VF test points showing inferior arcuate damage; C. RNFL clock hour meridian showing superotemporal thinning; D. Enface and thickness map showing superotemporal thinning; E. Visual field showing the inferior arcuate defect and nasal step; F. GCL thickness map showing an unspecified pattern. GCL — probability VF test points showing an unspecified pattern

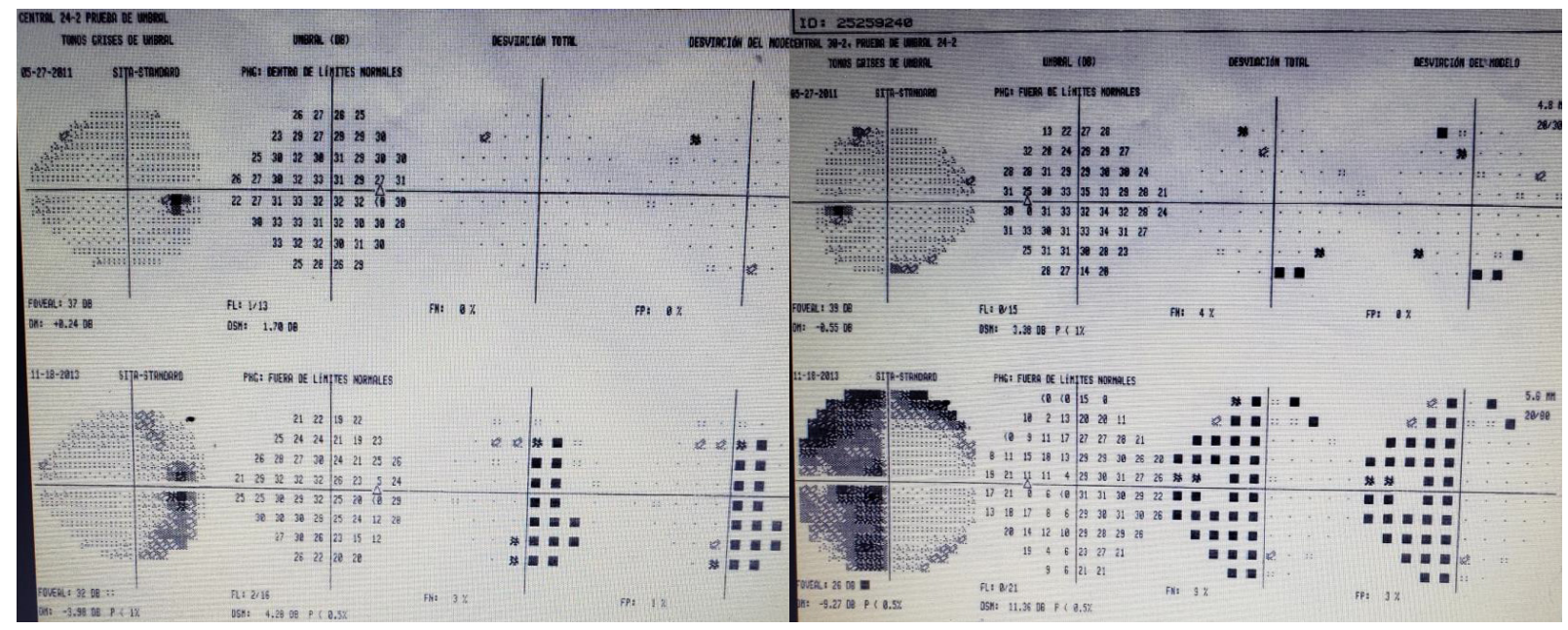

FIGURE 5. The visual field findings in 2013 when the craniopharyngioma was diagnosed for the first time. At this time, the defects were completely different from the actual presentation in the left eye. In 2013, the right eye showed a similar nasal hemifield involvement. The left eye showed a temporal hemifield defect as opposed to the actual presentation, where it showed an inferior arcuate defect. 2011 visual fields showed no visual field compromise in both eyes, as opposed to 2013 visual fields hemifield compromise mentioned previousl

of the RNFL compared with the vertical portion of the optic disc [8]. On the other hand, the pattern in glaucoma affects the vertical portion (superior and inferior quadrants) of the optic disc [9]. Patients with bitemporal hemianopsia have demonstrated reduced RNFL thickness measured by OCT compared with healthy controls [10].

The fovea, which is the center of the visual field, is measured with field macular GCC analysis, and it can easily be correlated with the visual field defect. 
Patients with bitemporal visual field loss from chiasmal compression have shown a better correlation with macular GCC than RNFL measurements [11]. Patients with chiasmal compression have a greater degree of thinning in the nasal macula than healthy controls. Due to the disruption of the crossing fibers originating in the nasal retina, these findings explain this pattern $[12,13]$

In patients with compressive lesions of the anterior visual pathways, there is a correlation between preoperative macular GCC thickness and postoperative visual outcome. In a study of twenty-three patients with chiasmal compression imaged with the cirrus high-definition optical coherence tomography macular cube, RNFL scan protocols, and automated (30-2 Humphrey) visual fields, authors concluded that patients with better postoperative 30-2 Humphrey visual fields had greater preoperative macular GCC thickness [14]. Pre- and postoperative RNFL and macular GCC to assess the postoperative follow-up are the guidelines for patients with pituitary adenomas recommended by The Congress of Neurological Surgeons [15].

After surgery, in patients with RNFL and macular GCC, the thinning may persist or even worsen despite having normal visual fields [16]. Several patients may appear to have a "paradoxical" worsening of the OCT measurements coincident with the improvement of the visual function because it takes at least six weeks for retrograde degeneration to be complete. After removal of the conduction block followed by secondary remyelination and restoration of axoplasmic flow, visual recovery occurs in stages months to years after surgery $[17,18]$. Future presentations of recurring compression may manifest first as progressive thinning of the macular GCC and RNFL.

The discovery of incidental mass lesions in close proximity to the visual pathways is not uncommon because of the frequent use of neuroimaging in clinical practice. In a study of forty patients undergoing surgical resection of para-chiasmal lesions, patients were prospectively assessed before surgery with a complete neuro-ophthalmic examination, including standard automated visual field (VF) testing and OCT measurements of RNFL thickness. It was found that $15 \%$ of patients had thin RNFLs in the presence of normal visual field testing. Patients with compressive optic neuropathies can present damage to the anterior visual pathways before visual field loss occurs [19].
In a study of 23 patients (46 eyes) with pituitary adenomas, 12 eyes had normal visual fields, and 34 eyes had visual field defects. Authors concluded that the preperimetric group had normal RNFL thickness but significantly reduced OCT macular GCC thickness compared with healthy controls. They emphasize the utility of OCT of the macular GCC in the evaluation of these patients because nasal thinning of the macular GCC was found to be a better predictor than RNFL parameters in detecting chiasmal compression [20].

\section{CONCLUSIONS}

Optical coherence tomography is a routine non-invasive imaging device in ophthalmology. It is in evaluating patients with compressive lesions of the anterior visual pathways. The RNFL and macular GCC thickness can help differentiate compressive optic neuropathies from other neuropathies, including glaucoma.

In this case, in particular, OCT findings showed a high correlation with visual field defect and contributed to a correct diagnosis.

\section{REFERENCES}

1. Hoyt WF, Luis 0 . The primate chiasm. Details of visual fiber organization studied by silver impregnation techniques. Arch Ophthalmol. 1963; 70: 69-85, doi: 10.1001/archopht.1963.00960050071013, indexed in Pubmed: 13955217.

2. Huang $D$, Swanson $E A$, Lin $C P$, et al. Optical coherence tomography. Science. 1991; 254(5035): 1178-1181, doi: 10.1126/science.1957169, indexed in Pubmed: 1957169.

3. Popescu DP, Choo-Smith LP, Flueraru C, et al. Optical coherence tomography: fundamental principles, instrumental designs and biomedical applications. Biophys Rev. 2011;3(3): 155, doi: 10.1007/s12551-0110054-7, indexed in Pubmed: 28510064.

4. Micieli JA, Newman NJ, Biousse V. The role of optical coherence tomography in the evaluation of compressive optic neuropathies. Curr Opin Neurol. 2019; 32(1): 115-123, doi: 10.1097/WC0.0000000000000636, indexed in Pubmed: 30418197.

5. Bussel II, Wollstein G, Schuman JS. OCT for glaucoma diagnosis, screening and detection of glaucoma progression. $\mathrm{Br} \mathrm{J}$ Ophthalmol. 2014; 98(Suppl 2): ii15-ii19, doi: 10.1136/bjophthalmol-2013-304326, indexed in Pubmed: 24357497.

6. Al-Dahmani K, Mohammad S, Imran F, et al. Sellar Masses: An Epidemiological Study. Can J Neurol Sci. 2016; 43(2): 291-297, doi: 10.1017/cjn.2015.301, indexed in Pubmed: 26522017.

7. Biousse V, Newman N. Diagnosis and clinical features of common optic neuropathies. Lancet Neurol. 2016; 15(13): 1355-1367, doi: 10.1016/s1474-4422(16)30237-x, indexed in Pubmed: 27839652.

8. Unsöld R, Hoyt WF. Band atrophy of the optic nerve. The histology of temporal hemianopsia. Arch Ophthalmol. 1980; 98(9): 1637-1638, doi: 10.1001/archopht.1980.01020040489020, indexed in Pubmed: 7425927.

9. Mwanza JC, Oakley JD, Budenz DL, et al. Cirrus Optical Coherence Tomography Normative Database Study Group. Ability of cirrus HD-OCT optic nerve head parameters to discriminate normal from glaucomatous eyes. Ophthalmology. 2011; 118(2): 241-248.e1, doi: 10.1016/j. ophtha.2010.06.036, indexed in Pubmed: 20920824.

10. Sun $M$, Zhang Z, Ma C, et al. Quantitative analysis of retinal layers on three-dimensional spectral-domain optical coherence tomography for 
pituitary adenoma. PLoS One. 2017; 12(6): e0179532, doi: 10.1371/ journal.pone.0179532, indexed in Pubmed: 28628662.

11. Akashi A, Kanamori A, Ueda K, et al. The detection of macular analysis by SD-OCT for optic chiasmal compression neuropathy and nasotemporal overlap. Invest Ophthalmol Vis Sci. 2014; 55(7): 4667-4672, doi: 10.1167/iovs. 14-14766, indexed in Pubmed: 25015351.

12. Zehnder S, Wildberger H, Hanson JVM, et al. Retinal Ganglion Cell Topography in Patients With Visual Pathway Pathology. J Neuroophthalmol. 2018; 38(2): 172-178, doi: 10.1097/WN0.0000000000000589, indexed in Pubmed: 29210928.

13. Monteiro MLR, Hokazono K, Fernandes DB, et al. Evaluation of inner retinal layers in eyes with temporal hemianopic visual loss from chiasmal compression using optical coherence tomography. Invest Ophthalmol Vis Sci. 2014; 55(5): 3328-3336, doi: 10.1167/iovs.1414118, indexed in Pubmed: 24764062.

14. Tieger MG, Hedges TR, Ho J, et al. Ganglion Cell Complex Loss in Chiasmal Compression by Brain Tumors. J Neuroophthalmol. 2017; 37(1): 7-12, doi: 10.1097/WN0.0000000000000424, indexed in Pubmed: 28192385

15. Newman S, Turbin R, Bodach M, et al. Congress of Neurological Surgeons Systematic Review and Evidence-Based Guideline on Pretreatment Ophthalmology Evaluation in Patients With Suspected Nonfunctioning Pituitary Adenomas. Neurosurgery. 2016; 79(4):
E530-E532, doi: 10.1227/neu.0000000000001388, indexed in Pubmed: 27635960.

16. Danesh-Meyer HV, Wong A, Papchenko T, et al. Optical coherence tomography predicts visual outcome for pituitary tumors. J Clin Neurosci. 2015; 22(7): 1098-1104, doi: 10.1016/j.jocn.2015.02.001, indexed in Pubmed: 25891894.

17. Moon $\mathrm{CH}$, Hwang SC, $\mathrm{Ohn} \mathrm{YH}$, et al. The time course of visual field recovery and changes of retinal ganglion cells after optic chiasmal decompression. Invest Ophthalmol Vis Sci. 2011; 52(11): 7966-7973, doi: 10.1167/iovs.11-7450, indexed in Pubmed: 21896856.

18. Cottee L, Daniel C, Loh W, et al. Remyelination and recovery of conduction in cat optic nerve after demyelination by pressure. Exp Neurol. 2003; 184(2): 865-877, doi: 10.1016/s0014-4886(03)00310-8, indexed in Pubmed: 14769379.

19. Danesh-Meyer HV, Papchenko T, Savino PJ, et al. In vivo retinal nerve fiber layer thickness measured by optical coherence tomography predicts visual recovery after surgery for parachiasmal tumors. Invest Ophthalmol Vis Sci. 2008; 49(5): 1879-1885, doi: 10.1167/iovs.071127, indexed in Pubmed: 18263812.

20. Yum HRi, Park SH, Park HYL, et al. Macular Ganglion Cell Analysis Determined by Cirrus HD Optical Coherence Tomography for Early Detecting Chiasmal Compression. PLoS One. 2016; 11(4): e0153064, doi: 10.1371/journal.pone.0153064, indexed in Pubmed: 27049647. 